\title{
Complex method of defects diagnostics in underground structures
}

\author{
Alexey Beskopylny ${ }^{1, *}$, Alexander Lyapin ${ }^{1}$, Maxim Kadomtsev $^{1}$, and Andrey Veremeenko ${ }^{1}$ \\ ${ }^{1}$ Don State Technical University, pl. Gagarin, 1, Rostov-on-Don, 344010, Russia
}

\begin{abstract}
The article presents a comprehensive method for diagnosing underground structures using the example of an underground pedestrian crossing located in Rostov-on-Don. The problem of assessing the condition of buildings and structures is very relevant at all stages of the life cycle. There is a special need for continuous monitoring of bearing structures for many buildings with critical applications especially if reliability of which determines the life and health of people. This work considered complex method included geodetic research, geological study of the state of subsoil, analysis of state of steel and reinforced concrete structures, vibro diagnostics of load-bearing structures under dynamic technogenic impacts and an assessment of the mechanical characteristics of steel and reinforced concrete structures in order to develop a conclusion on the overall state of the transition and the possibility of its further operation. Analysis of the response from arbitrary non-stationary effects on structural elements is carried out on the base of the calculated spectrum of reference impacts.
\end{abstract}

\section{Introduction}

Recently the problem of assessing the condition and working capacity of buildings and structures becomes increasingly important. At the same time, the need for technical survey not depends on the phase of construction and building purpose. Modern trends in construction, such as the increase in the number of storeys of buildings, the compaction of urban buildings, the tightness of construction sites, the development of underground space, and the saturation with engineering communications invariably lead to the emergence and subsequent increase in the negative techno genic impact of the construction carried out on already constructed facilities located in adjacent areas. So the special importance of the problem of monitoring the technical condition of buildings and structures is become in order to prevent the emergence. It is obvious that the assessment of the technical state of load-bearing structures should be systematic and allow for the evaluation of the changes taking place on the basis of quantitative criteria, based on procedures to identify the compliance of the actual strength, rigidity and stability of structural elements to regulatory requirements.

There are many methods of mechanical properties assessment that are useful for monitoring. Depending on the building purpose the methods to determine the structures

${ }^{*}$ Corresponding author: besk-an@ya.ru 
properties are different. These are non-destructive tests based on indentation methods that used for metal structures [1,2], determination of the rheological parameters for the creep models [3], identification of the structures properties [4].

Complex and multidisciplinary approach to the diagnosis of historical buildings is shown in [5]. Items related to various issues as structural and thermal aspects, acoustic requirements and efficiency of active energy systems are took into account.

Damages and defects happening throughout structure execution or transport and consignment on bridge structure are considered in [6]. The research represents an automated laser remote sensing data with Bridge Information Model for a constructed bridge.

A case study of different approaches to planning and building control focus on physical and technical issues and on design and construction and in a social and environmental context at the other hand is in [7]. This paper suggests thinking and acting in terms of complex ecosystems and addressing the issues from an anthropological perspective.

The defects, faults and accidents of contemporary constructions are considered in [8]. The article focuses on the defects and disruptions of buildings, which caused death or serious injuries (human or animal), or major property damage.

All cases show that a complex assessment of the state of a building is divided into a study of the state of building elements, their strength and other physical characteristics.

\section{Problem statement and equipment}

The objectives of the survey included:

- Preliminary (visual inspection) with the registration of damages and drawing up of the defective sheet;

- Carrying out geodetic measuring works, deviations from axes, deplanation;

- Non-destructive testing of the strength of structural elements;

- Non-destructive testing of strength of steel structures, reinforcement bars;

- Laboratory evaluation of strength of elements of stone structures on the basis of destructive methods;

- Geological analysis of soil in which there are buildings;

- Study of the effect of vibration exposure on the bearing capacity transitions from passing over them transport;

- Analysis of the causes of defects in structures;

- Development of conclusions about the technical condition of building structures, pedestrian subways.

Tests of monolithic reinforced concrete structures made by the method of surface ultrasonic testing according to standard methods. The strength of concrete in monolithic reinforced concrete structures is determined by an ultrasonic method for the calibration dependence, built in accordance with the requirements of MDS 62-2.01 according to parallel tests of the same sections of concrete structures by ultrasonic method (GOST 17624-87) and by the method of separation with shear fracture (GOST 22690-88).

During test of steel structural elements there are objective difficulties, the main of which are the following. To carry out the standard test it needs to cut out segments of the exploited construction material, and to make specimen standard sizes. The greatest interest is the most loaded place where the weakening of the cross section cut is associated with maximum risk of destruction of the structure itself. If you consider that obtaining reliable information is possible only on the basis of the processing is quite a representative sample (10-15 samples of elements of the same grade of steel), these difficulties become even more obvious. Furthermore, during prolonged operation the metal characteristics change from the 
viewpoint of strength and plastic, and reduce their carrying capacity, causing even greater doubts about the appropriateness of carrying out destructive tests.

In this regard, we have developed a unique system of non-destructive control of strength of metal, which to date has tens of approbation under the control of the mechanical characteristics of responsible metal structures and allows to simultaneously determine the main mechanical properties: hardness, yield strength, tensile strength, elongation at any place design. The instrument [9] for determining the mechanical properties of steels (Fig. 1), which consist of handle 1, spring 2, striker 3, body 4, indenter holder 5, sensor 6 and 9 , body part 7 , damper 8 .

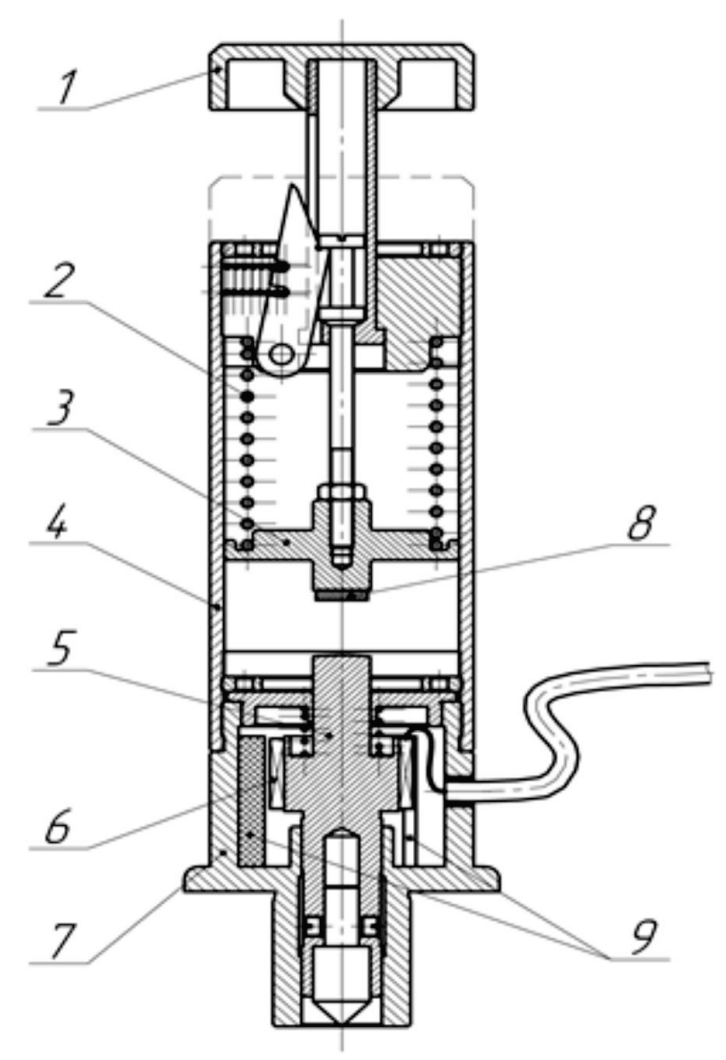

Fig. 1. The devise for mechanical properties measurement.

During measurement striker 3 hits over the indenter holder 5 and sensor 6 registers signal of velocity $V(t)$. The dependences $S(t)$ and $W(t)$ are obtained by integrating and differentiating [10].

\section{Investigation of dynamic effects on the structure of the pedestrian crossing when driving a motor vehicle}

Vibration measuring 12-channel device VK-12 (Fig. 2) is developed and implemented on the basis of the E14-440 module manufactured by the company "L-Card" and is designed for receiving, amplifying and converting mechanical oscillations of the elements of the structure under dynamic influences, Including at the noise level (microseismic vibrations) and signal transmission in digital form for further processing in IBM PC. 
During the experimental studies accelerometer sensors were fixed in the centre of the ceiling panel with the help of anchor bolts. This type of fastening provides the best matching of the sensor and over covering.

In the course of experimental studies, the level of vibration velocities from the following sources was investigated:

- the impact of a car with a mass of 20.5 tons at a speed of $30 \mathrm{~km} / \mathrm{h}$ and $50 \mathrm{~km} / \mathrm{h}$;

- hit on the supporting beam.

For experimental determination of the frequencies of the Flexural vibrations of a beam was used the results of the registration of the accelerations of vibrations under the impact. The impact corresponds to a Gaussian pulse

$$
p(t)=P e^{-a^{2} t^{2}}
$$

where $P$ - the peak value of impact load, 2/a-time pulse.
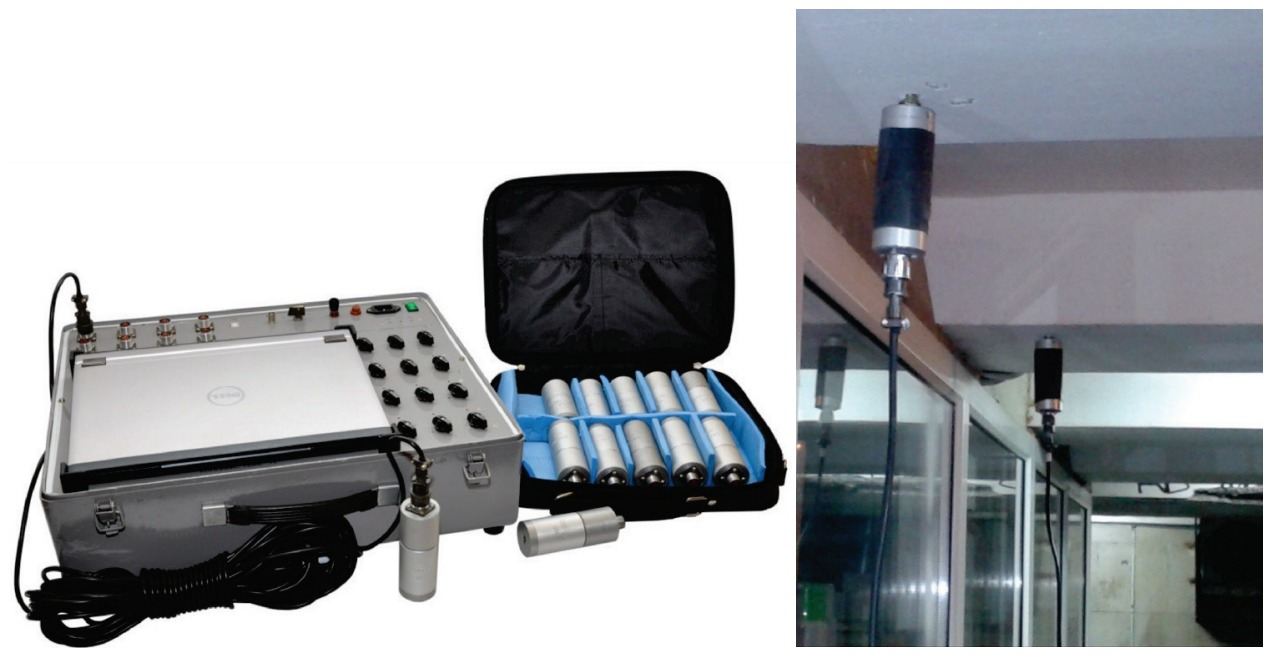

Fig. 2. Mobile vibro-measuring complex.

The spectrum of the Gaussian pulse is defined by the formula

$$
S(\omega)=P \int_{-\infty}^{\infty} e^{-a^{2} t^{2}} e^{-i \omega t} d t=\frac{P \sqrt{\pi}}{a} e^{-\left(\frac{\omega}{2 a}\right)^{2}},
$$

where $\omega=2 \pi f$ - circular frequency of oscillation, $f$ is the linear frequency.

With an external effect on the structure, the spectrum of the detected response depends on the spectrum of the external action and the spectral characteristics of the structure. This dependence is expressed by the formula

$$
S_{o}=S_{b} \cdot S_{\mathrm{K}}
$$

Where $S_{0}$ is the response spectrum, $\mathrm{S}_{b}$ is the external impact spectrum, $S_{\kappa}$ is spectrum of natural frequencies of the structure. Then the spectrum of the construction is determined by the formula

$$
S_{\mathrm{K}}=\frac{S_{\mathrm{O}}}{S_{\mathrm{B}}}
$$


Thus, it follows from (4) that under impact conditions the response spectrum will differ very little from the intrinsic spectrum of the structure. To determine the dynamic response to an arbitrary dynamic effect, it is necessary:

1. Perform a test record of the response to the dynamic impact in question at an arbitrary location (for example, at a low-intensity auto-horn).

2. Perform the same registration of the response to the reference impact.

3. Get the response of the structure by the formula

$$
V(t)=\frac{1}{2 \pi} \int_{-\infty}^{\infty} \frac{\overline{V_{n c}}(\omega)}{\overline{V_{s c}}(\omega)} \cdot \overline{V_{s}}(\omega) \cdot e^{i \omega t} d t,
$$

Where $\overline{V_{s c}}(\omega)$ is the response velocity spectrum for the standard effect, $\overline{V_{n c}}(\omega)$ is the response velocity spectrum for the nonstationary effect under study, $\bar{V}_{s}(\omega)$ is the velocity spectrum of the response to the reference effect recorded on the elements of the pedestrian crossing structure.

\section{Results and discussion}

Visual inspection was conducted with the establishment of damages and drawing up of a defective sheet. As a result, a set of cracks with a width of 0.3 to $1 \mathrm{~mm}$ was revealed, several traces of soaking, several exposures of working reinforcement of reinforced concrete structures, recommendations for restoration.

When geodetic work on each of the tunnels, the leveling of the ceiling was leveled. For branched underground pedestrian crossings, the rooms were further divided into sectors. The work was carried out by the method of geometric leveling, using the leveling GEOBOX N7-26. Leveling was carried out on the transverse beams, each of which was taken at three points

The non-destructive testing of steel strength was subjected to armature elements of the reinforcement of the slabs of the coating. A grinding machine stripped the armature metal and the mechanical properties were determined using the "Strength" system: hardness (HB), yield stress $\left(\sigma_{y}\right)$, tensile strength $\left(\sigma_{b}\right)$ and elongation $(\delta)$. The survey showed that for the wall panel reinforcement the average values of $\mathrm{HB}=150, \sigma_{y}=344 \mathrm{MPa}, \sigma_{b}=437$ $\mathrm{MPa}, \delta=23 \%$ correspond to the requirements for the strength class of the A400 armature (AII).The strength characteristics obtained correspond to the requirements for the strength of reinforcing steels specified in the standard structure.

To determine the spectral characteristics of the response of structural elements to the dynamic effects from the transport passage 3 sensors accelerometers were installed in the zones with the greatest load of the beam.

Analysis of the response to impact showed that a resonance peak occursat a frequency of $45 \mathrm{~Hz}$ (Fig. 3). This result is in good agreement with the results obtained from the experimental full-scale studies of the oscillations of ceiling panel under the action of a shock load. It should be noted that the resonance obtained as a result of the calculation is somewhat lower than that obtained experimentally. Hence it can be concluded that in panel on which the registration was carried out, there were no processes that could worsen the dynamic properties of the panel. 


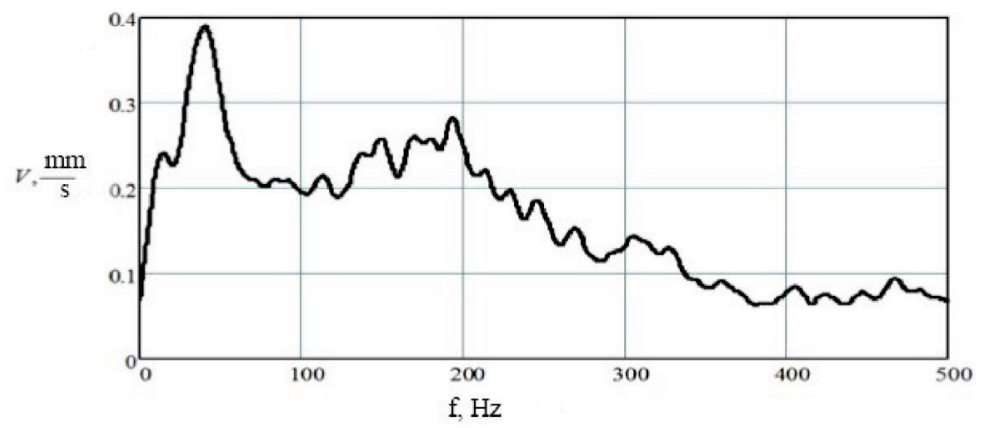

Fig.3. The amplitude-frequency characteristics of vibration velocities of the response of the slab.

\section{Conclusions}

As a result of the studies, the following conclusions can be drawn:

- maximum dynamic stresses from the impact of individual units of passing vehicles do not exceed $15 \mathrm{kPa}$;

- Total stresses when passing vehicles do not exceed $16 \mathrm{MPa}$

- the maximum speed of ceiling panel from the impact of individual units of passing vehicles did not exceed $0.4 \mathrm{~mm} / \mathrm{s}$;

- under the existing speed limits, the greatest dynamic stresses under the influence of the machine flow will not exceed $30 \mathrm{kPa}$;

- frequency spectrum of impact from passing vehicles is within the limits of 1-20 Hz;

- the intrinsic frequency of bending oscillations of the panel is about $40 \mathrm{~Hz}$.

- the natural frequency of the panel lies outside the range of frequencies that are excited in the floors when driving. Due to this, there will be no resonance of panel during the passage of vehicles

Studies have shown that the transition is in a workable state. Required activities:

A) in accordance with the defective sheet, to strengthen the elements of the entrance design according to a specially executed project;

B) in places of strong soaking, dismantle the lining, inspect the technical condition of the panels and perform the necessary repairs;

C) in the zone where the reinforcement is exposed, clean the reinforcement with metal brushes and repair the protective layer of concrete with a repair composition;

D) carry out work to eliminate the flow of rainwater;

E) in the places where the cracks are opened, install beacons.

Thus, a comprehensive method for diagnosing defects in underground structures has been developed, including a number of authoring techniques for vibration diagnostics of the dynamic characteristics of structural elements, determining their resonant frequencies and the complex of mechanical characteristics of steel bearing structures.

\section{References}

1. A.N. Beskopylny, A.A. Veremeenko, B.M. Yazyev, MATEC Web of Conferences 106, 04004 (2017)

2. A.N. Beskopylny, I.G. Kadomtsev, N.I. Beskopylnaya, Theoretical foundations of civil engineering 83-84 (2005)

3. A.S. Chepurnenko, V.I. Andreev, B.M. Yazyev, A.N. Beskopylny, MATEC Web of Conferences 67, 06059 (2016) 
4. A.N. Beskopylny, A.A. Lyapin, V.I. Andreev MATEC Web of Conferences 117, 00018 (2017)

5. F. Ascione, F. Ceroni, R. F. De Masi, F. De'Rossi, M.R. Pecce, Applied Energy 185 Part 2, 1517-1528 (2017)

6. M. Sedec, A. Serwa, The Egyptian Journal of Remote Sensing and Space Science 19, issue 2, 273-283 (2016)

7. K. Alexander, Procedia Engineering 20,1-11 (2011)

8. M. Kraus, T. Vondráčková, V. Nývlt, MATEC Web of Conferences 93, 03004 (2017)

9. D.M. Belenkii, A.N. Beskopylny, N.N. Beskopylnyi, E.K. Polibin, B.A. Pesenko Method for determining mechanical characteristics and the device for its implementation. Patent No. 2079831 (1997)

10. D.M. Belenkii, A.N. Beskopylnyi, N.L., L.G. Shamraev, Industrial laboratory 64 (5), 340-343 (1998) 
